\title{
Revealing the microbial assemblage structure in the human gut microbiome using latent Dirichlet allocation
}

\author{
Shion Hosoda ${ }^{1,2}$, Suguru Nishijima ${ }^{1,2,3}$, Tsukasa Fukunaga ${ }^{1,4}$, Masahira Hattori ${ }^{1,3,5}$ and Michiaki \\ Hamada $1,2,6,7^{*}$ (if
}

\begin{abstract}
Background: The human gut microbiome has been suggested to affect human health and thus has received considerable attention. To clarify the structure of the human gut microbiome, clustering methods are frequently applied to human gut taxonomic profiles. Enterotypes, i.e., clusters of individuals with similar microbiome composition, are well-studied and characterized. However, only a few detailed studies on assemblages, i.e., clusters of co-occurring bacterial taxa, have been conducted. Particularly, the relationship between the enterotype and assemblage is not well-understood.
\end{abstract}

Results: In this study, we detected gut microbiome assemblages using a latent Dirichlet allocation (LDA) method. We applied LDA to a large-scale human gut metagenome dataset and found that a 4-assemblage LDA model could represent relationships between enterotypes and assemblages with high interpretability. This model indicated that each individual tends to have several assemblages, three of which corresponded to the three classically recognized enterotypes. Conversely, the fourth assemblage corresponded to no enterotypes and emerged in all enterotypes. Interestingly, the dominant genera of this assemblage (Clostridium, Eubacterium, Faecalibacterium, Roseburia, Coprococcus, and Butyrivibrio) included butyrate-producing species such as Faecalibacterium prausnitzii. Indeed, the fourth assemblage significantly positively correlated with three butyrate-producing functions.

Conclusions: We conducted an assemblage analysis on a large-scale human gut metagenome dataset using LDA. The present study revealed that there is an enterotype-independent assemblage.

Keywords: Metagenomics, Latent Dirichlet allocation, Human gut microbiome, Enterotype, Microbial assemblage, Bayesian model, Machine learning

\section{Introduction}

The human gut microbiome varies greatly from person to person depending on differences among human populations [1] and dietary habits [2]. The differences in gut microbial compositions affect host health and physiology [3], and in some cases, altered microbial compositions are

\section{*Correspondence: mhamada@waseda.jp}

${ }^{1}$ Graduate School of Advanced Science and Engineering, Waseda University, 55N-06-10, 3-4-1, Okubo Shinjuku-ku, 169-8555 Tokyo, Japan

${ }^{2}$ Computational Bio Big-Data Open Innovation Laboratory (CBBD-OIL), National Institute of Advanced Industrial Science and Technology (AIST), Tokyo, Japan Full list of author information is available at the end of the article associated with diseases, such as inflammatory bowel disease (IBD) [4], type 1 diabetes [5], colorectal cancer [6], and autism [7, 8]. Recent developments in metagenome sequencing technologies have enabled investigations of gut microbial compositions of individuals with ease and rapidity, and many large-scale research projects focused on the human gut microbiome have been conducted $[1,9$ 11]. At present, by applying various data mining methods to these massive metagenomic datasets, the structure of the human gut microbiome and the relationship between a hosts phenotype and its gut microbial profile can be revealed. 
Cluster analysis of samples is one of the widely used data-mining methods in metagenomic research. With this approach, individuals are clustered into groups based on similarities in their microbial profiles, that is, each sample is assigned to one cluster by this method. For example, Arumugam et al. discovered that the gut microbial profiles of individuals could be classified into three types known as enterotypes using the partitioning around medoids (PAM) clustering method [12]. In another example, Ding and Schloss reported, employing the Dirichlet multinomial mixture (DMM) clustering method, that the human gut microbiome has considerable inter-individual variation and that the cluster type of an individual was almost unchanged during the sampling period [13, 14]. Although cluster analysis is a powerful approach for uncovering the overall structure of the human gut microbiome, this analysis is strongly affected by the dominant microbes in each individual. Therefore, cluster analyses of samples may ignore the existence of non-dominant but shared microbes among individuals (Fig. 1).

An alternative data-mining method is microbial assemblage analysis, which clusters microbes into certain assemblages, instead of clustering samples into groups. Here, following Shafiei et al. [15], we define microbial assemblages as groups of microbes that are expected to co-occur. The existence of microbial assemblages can be reasonably expected from the interactions between microbes [16]. Several microbial assemblages can exist in one individual, and microbial assemblage analysis can capture assemblages consisting of non-dominant microbes, unlike a cluster analysis of samples (Fig. 1). Shafiei et al. developed BioMiCo, which is a Bayesian probabilistic model for microbial assemblage analysis, and discovered host-specific assemblages in human gut metagenomic time-series data [15]. Cai et al. also explored microbial assemblages using non-negative matrix factorization methods and identified a shift of microbial assemblages through time in one individual [17]. Higashi et al. developed Latent Environment Allocation, a web application for visualization of metagenomic data based on a microbial assemblage analysis method, and found that microbial assemblages can represent continuous variations of the human gut microbiome [18]. Meanwhile, Yan et al. created MetaTopics, an $\mathrm{R}$ package for microbial assemblage analysis [19]. Further, Sankaran and Holmes conducted a simulation study to compare several methods of microbial assemblage analyses [20]. Microbial assemblage analysis of the human gut microbiome has also been applied to track sources of contamination in metagenomic research [21] and detect assemblage-level metabolic interactions [22]. Although many microbial assemblage analyses of the human gut microbiome have been performed, a comparison between classical enterotypes and the assemblages of co-occurring taxa has not yet been conducted. Consequently, the large-scale assemblage structure of the human gut microbiome and the relationship between microbial assemblages and enterotypes are still unclear.

In this study, we carried out a microbial assemblage analysis of a large-scale human gut metagenomic dataset to establish the relationship between microbial assemblages and enterotypes. To detect assemblages, we used the latent Dirichlet allocation (LDA) method, which is an unsupervised probabilistic model [23]; LDA was first proposed for the classification of documents in naturallanguage processing, and this method is now widely applied in bioinformatics fields, such as transcriptome analysis [24], pharmacology [25], gene function prediction [26], and metagenomic analyses [18-20, 27]. We first investigated the number of microbial assemblages based on the relationship between microbial assemblages
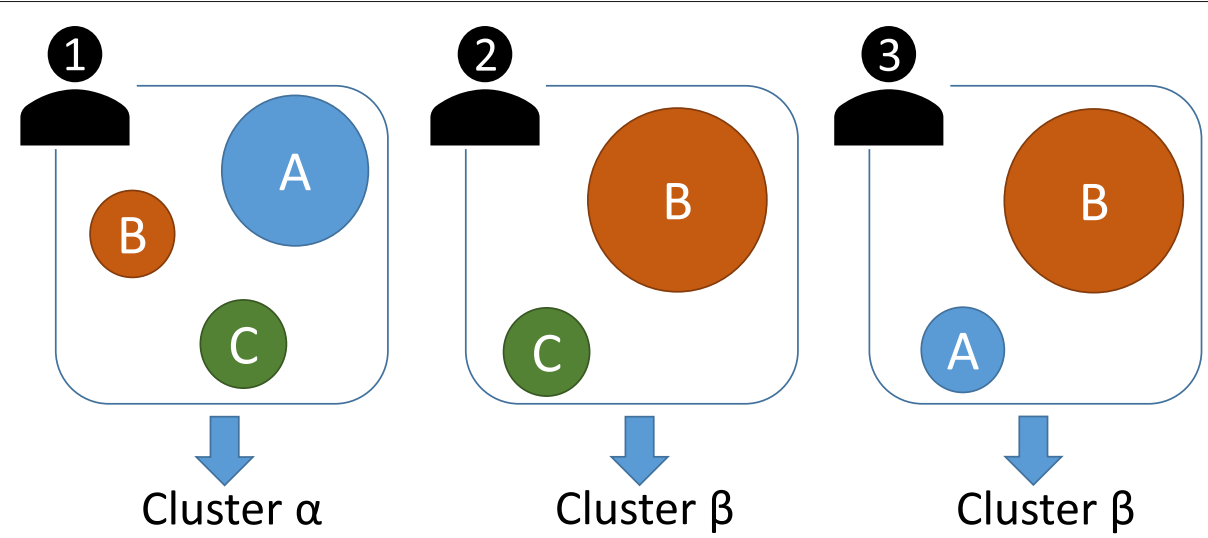

Fig. 1 Schematic illustration of microbial assemblages and cluster analysis for the human gut microbiome, where A, B, and C indicate microbial assemblages, with circle size indicating abundance. The cluster of each individual is determined by the dominant assemblage. However, a cluster analysis cannot capture the non-dominant but shared microbes among samples, such as those comprising assemblage $\mathrm{C}$ 
and enterotypes. We found that a 4-assemblage model has high interpretability in the context of a large-scale human gut microbiome dataset and discovered that an individual might have not just one microbial assemblage but several assemblages in many cases. We investigated the relationships between enterotypes and microbial assemblages and revealed that three of the assemblages could be matched to the three enterotypes; however, the fourth assemblage could exist in all enterotypes. In addition, the dominant genera of this assemblage included butyrate-producing species, and this assemblage is significantly positively correlated with three butyrate-producing functions. We also estimated the functions of each assemblage by applying LDA to the functional profiles of the same samples and found that the fourth assemblage has some specific functional categories, such as the immune system and translation, with high abundance.

\section{Materials and methods}

We used our own implementation of the LDA and PAM algorithms. The detailed information is described in "Availability of data and material."

\section{Metagenomic dataset and preprocessing methods}

We used the large-scale human gut metagenome dataset constructed by Nishijima et al. [28]. This dataset consisted of gut metagenomic data from 861 healthy adults from 12 countries. Each individual corresponds to one sample. The taxon of each sequencing read was assigned by mapping the read to a reference genome dataset consisting of 6149 microbial genomes. We used genus as the taxonomic rank for each sequencing read, as commonly performed in previous studies on enterotypes [12-14, 29].

The read count of each genus of each individual was divided by the total read count of each individual, then multiplied by 10,000 and rounded down to the nearest integer. This is because the estimation results of LDA are strongly affected by samples with high read counts when using read count data directly. Therefore, we normalized each sample to sum to the same constant (that is, 10,000) to remove the bias caused by the differences of read counts among samples. We confirmed that the estimated parameters do not depend on the constant (Additional File 1: Figure S1). After these preprocessing steps, the number of different genera included in the dataset became 252 .

To calculate correlation coefficients between microbial assemblages and functions across individuals, we used the Kyoto Encyclopedia of Genes and Genomes (KEGG) [30] orthology-based annotated data as functional profiles. These functional profiles are of the same samples as the genus data.

\section{PAM clustering method}

To assign enterotypes, we applied the PAM clustering method to the dataset according to the methods of Arumugam et al. [12]. This algorithm clusters samples by iteratively updating each cluster's medoid, which is defined as the sample in a cluster for which the sum of a dissimilarity to the other samples in the same cluster is the smallest. The algorithm consists of the following three steps: (i) choose the initial value of the medoid randomly from the samples, (ii) assign each sample to the cluster with the smallest Jensen-Shannon divergence (JSD) to its medoid, and (iii) update the medoids using the JSD as the dissimilarity. Repeat steps (ii) and (iii) until the medoids no longer change. In the present study, we conducted 10 trials and used the result that had the highest silhouette coefficient [31].

\section{LDA for modeling the human gut microbiome}

The probabilistic LDA model [23] can be utilized to estimate $K$ microbial assemblages from a human gut metagenomic dataset, where $K$ is a given parameter. Let the numbers of individuals (samples) and genera be denoted by $N$ and $D$, respectively. In the LDA model, the $i$ th metagenome sample $(i \in\{1, \ldots, N\})$ has a categorical distribution with parameter $\theta_{i}=\left\{\theta_{i, k}\right\}_{k=1}^{K}$ over microbial assemblages where $\theta_{i, k}$ is the occurrence probability of the $k$ th assemblage in the $i$ th sample. The $k$ th microbial assemblage has a categorical distribution with parameter $\phi_{k}=\left\{\phi_{k, j}\right\}_{j=1}^{D}$ over genera, where $\phi_{k, j}$ is the occurrence probability of the $j$ th genus in the $k$ th assemblage.

A microbial assemblage with high probability in an individual implies that the individual tends to have that particular microbial assemblage in the gut microbiome, and a genus with high probability in a microbial assemblage indicates that the microbial assemblage tends to have that particular genus. In addition, the LDA model has prior distributions on $\theta_{i}$ and $\phi_{k}$ provided by the Dirichlet distribution with hyperparameters $\alpha$ and $\beta$, respectively. In this study, we used 0.1 and 0.05 as initial values for all the elements in $\alpha$ and $\beta$, respectively.

The LDA parameters $(\theta$ and $\phi)$ can be learned from the dataset in an unsupervised manner. Various parameter inference methods for the LDA model have been proposed, and we used the variational Bayes (VB) method [23]. The VB method maximizes an approximation of the marginal likelihood, called the variational lower bound (VLB) score, by updating the parameters iteratively from random initial values. We finished the iteration of the parameter update when the change in the VLB score between the previous and the current step was less than $10^{-6}$. Finally, we estimated each $\theta_{i}$ and $\phi_{k}$ as the expectation values of the posterior distribution estimated by the VB method. This parameter estimation method has been previously described by Asuncion et al. [32]. In addition, 
we updated the hyperparameters $\alpha$ and $\beta$ from the initial values using a fixed-point iteration method in the parameter learning step [33]. Based on previous research on LDA hyperparameter settings [34], we estimated the parameters such that each element of $\alpha$ differed from the others but all elements of $\beta$ had the same value. We conducted 10 trials for each $K=2,3,4$, and 5 and adopted the estimated set of parameters with the highest VLB score among all trials for each $K$.

\section{Functional assemblage analysis}

We estimated the functions of each assemblage by applying LDA to the functional profiles. We refer to the resulting assemblages as functional assemblages. Details surrounding the method are described in Section S1 (Additional File 1).

\section{Entropy scores of genera and individuals}

To quantify whether the estimated distributions are skewed toward some assemblages, we calculated the entropy scores of each sample and each genus over assemblages. In a categorical distribution, a high entropy score means that the distribution is similar to the uniform distribution, and a low score means that the distribution tends to take a specific value. The entropy score $H(i)$ of the $i$ th sample over assemblages was calculated as follows:

$$
H(i)=-\sum_{k=1}^{K} P(k \mid i) \log P(k \mid i) .
$$

As $P(k \mid i)$ is equal to $\theta_{i, k}$, we can directly calculate this score using the estimated LDA parameters. The entropy score, $H(j)$, of the $j$ th genus over assemblages was calculated as follows:

$$
\begin{aligned}
& H(j)=-\sum_{k=1}^{K} P(k \mid j) \log P(k \mid j), \\
& P(k \mid j)=\frac{P(j \mid k) P(k)}{\sum_{k} P(j \mid k) P(k)}
\end{aligned}
$$

where $P(j \mid k)$ is equal to $\phi_{k, j}$ and $P(k)$ was computed as the average of all $\theta_{i, k}$ across samples.

\section{Results}

Cluster analysis of the human gut microbiome enterotypes To investigate the relationship between enterotypes and assemblages, we classified individual samples into three clusters using the PAM clustering method. We observed that the dominant genera in each identified cluster were Bacteroides, Prevotella, and Blautia and that these genera were specific to each cluster (Additional File 1: Figure S2). These results were consistent with those of previous enterotype studies, in which the following three enterotypes were identified in the human gut microbiome: Bacteroides dominant type, Prevotella dominant type, and
Ruminococcus and Blautia dominant type [12]. Hence, we referred to these clusters as B-type, P-type, and R-type. These results were consistent across trials (Additional File 1: Figure S3)

\section{Analysis of the human gut microbial assemblage profiles estimated by LDA}

We estimated the $K$-assemblage LDA model parameters for $K=2,3,4$, and 5 to identify the model with the highest interpretability of relationships between enterotypes and assemblages. Figure 2 shows the assemblage distributions for each enterotype obtained by each model for each assemblage (the standard deviations of the distribution across individuals are shown in Additional File 1: Figure S4). The 2-assemblage model identified a B-type specific assemblage and a P- and R-type specific assemblage (IDs 1 and 2 in Fig. 2a). The 3-assemblage model estimated assemblages corresponding to each enterotype (Fig. 2b). In addition to these enterotype-specific assemblages, the 4- and 5-assemblage models inferred general assemblages that appear in all the enterotypes (Fig. 2cd). The strength of LDA is that it is possible to obtain such an assemblage. Adding a fifth assemblage is not informative because it yields two general assemblages (IDs 4 and 5 in Fig. 2d) that have the same abundance pattern for enterotypes. Therefore, we used the 4-assemblage model in this study. We emphasize that the existence of a general assemblage is not trivial in models with four or more assemblages because there are not always genera that appear in all enterotypes. These results are consistent across trials (Additional File 1: Figure S5)

In the following analysis, we call the assemblages with IDs 1, 2, and 3 the "B-assemblage," "P-assemblage," and "R-assemblage," respectively, because these assemblages appeared specifically in the B-, P-, and R-type individuals, respectively (Fig. 2c). In addition, we refer to the assemblage with ID 4 as the "C-assemblage" owing to the high proportion of Clostridium. Next, we investigated the taxonomic composition of each microbial assemblage. Figure 3 depicts the genus distribution of each microbial assemblage estimated by LDA (i.e., $\phi_{k}=\left\{\phi_{k, j}\right\}_{j=1}^{D}$ in the previous section). B- and P-assemblages mainly consisted of one dominant genus, Bacteroides and Prevotella, with relative frequencies of $71 \%$ and $66 \%$, respectively. Conversely, $\mathrm{R}$ - and $\mathrm{C}$-assemblages consisted of genera with moderate abundance. The genera that constituted the Rassemblage were Blautia (22\%), Bifidobacterium (20\%), and Ruminococcus (8.6\%). The C-assemblage consisted of Clostridium (18\%), Eubacterium (15\%), and unclassified Firmicutes (13\%).

[In the LDA model, a genus can appear in several microbial assemblages. We investigated whether genera occurred in just one specific assemblage or not using the entropy scores of genera over assemblages 

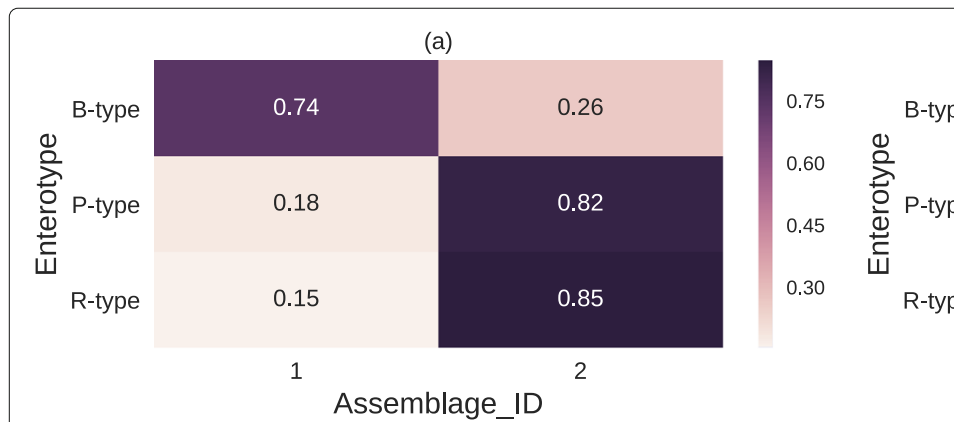

(c)

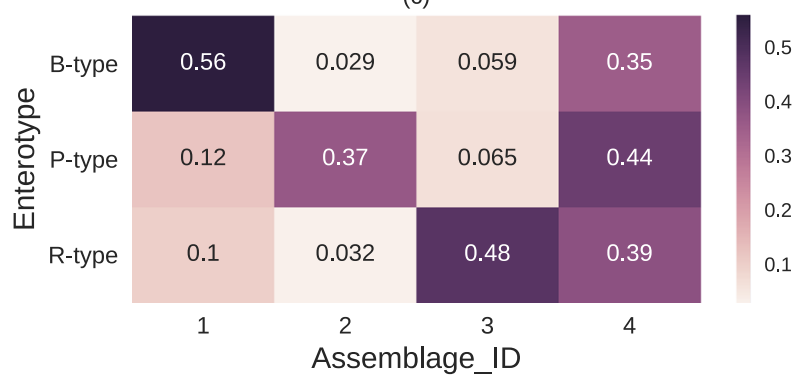

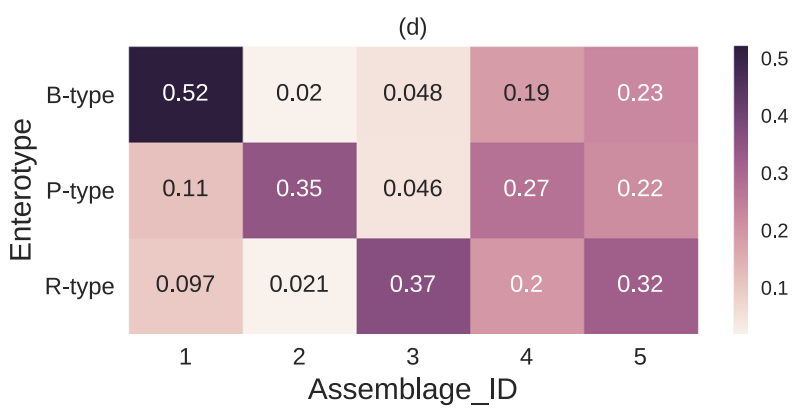

Fig. 2 Assemblage distributions for each enterotype. Each row shows a distribution obtained by averaging the estimated assemblage distributions across individuals of each enterotype. The $x$ - and $y$-axes represent the microbial assemblages and enterotypes, respectively. Darker colors indicate higher probabilities, and each number inside the partition indicates a different probability, where the sum of the values over each row is 1 . $\mathbf{a}$, $\mathbf{b}$, $\mathbf{c}$, and $\mathbf{d}$ indicate $K$-assemblage LDA models with $K=2,3,4$, and 5, respectively

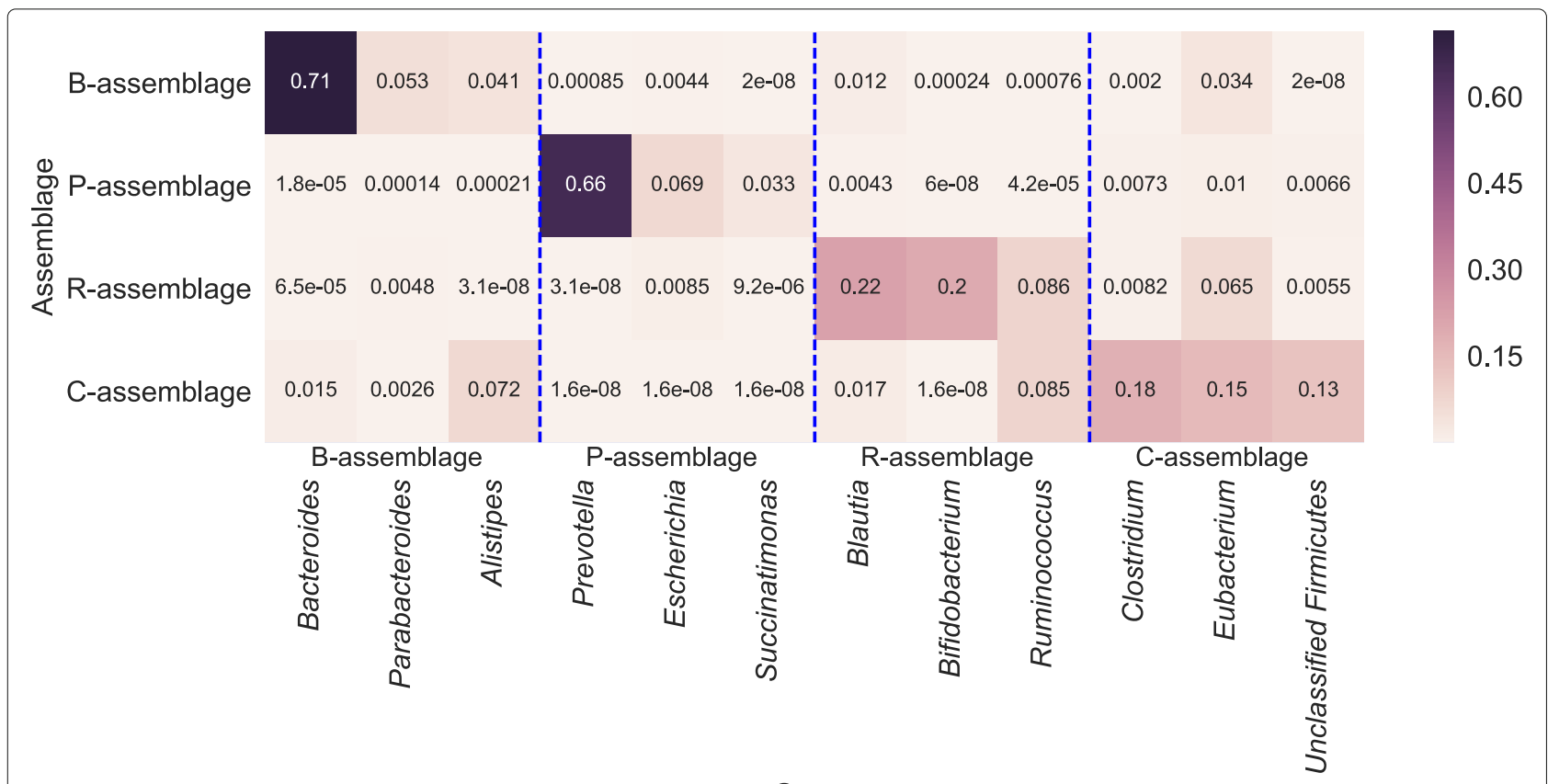

Genus

Fig. 3 Estimated genus distribution of each microbial assemblage. The $x$ - and $y$-axes represent genera and assemblages, respectively. We displayed only the three genera with the highest probability in each assemblage 
(Eq. 2). Figure 4a shows a histogram of the entropy scores for all genera, and two peaks at $0.00-0.125$ and $0.50-0.75$ can be observed within the distribution. The former peak represents assemblage-specific genera, and Bacteroides and Prevotella belonged to this group (Additional File 1: Table S1). The latter peak represents a genus appearing in several, but not all, assemblages, and Ruminococcus and Blautia belonged to this group (Additional File 1: Table S1). Several genera had high entropy scores, thereby indicating that they are universal genera among assemblages (Additional File 1: Figure S6).

Next, we calculated the entropy score of individuals over assemblages (Eq. 1). The distribution of the entropy scores was unimodal (Fig. 4b), and the median was 0.7805 . These results suggest that most individuals have multiple, but not all, microbial assemblages. In addition, we examined the distribution of assemblages within individuals (Additional File 1: Figure S7) and found that the co-abundance tendencies between microbial assemblages were not uniform. Individuals with $\mathrm{B}$-assemblage, $\mathrm{P}$-assemblage, $\mathrm{R}$ assemblage, and $\mathrm{C}$-assemblage dominance tend to have neither the $\mathrm{P}$ - nor R-assemblage, not the R-assemblage, not the $\mathrm{P}$-assemblage, and possess any other assemblages, respectively. All the individuals tend to have the $\mathrm{C}$-assemblage. These co-abundance tendencies can occur in the case that there are actually four enterotypes and one corresponding assemblage for each. To investigate such a possibility, we performed 4-type PAM clustering, but no such one-to-one relationship was observed (Additional File 1: Figure S8). Therefore, the C-assemblage can be regarded as an assemblage that appears in all three enterotypes.

\section{Relationships between microbial assemblages and countries}

We investigated the relationship between microbial assemblages and host countries. Figure 5 shows the average assemblage distributions of individuals for each country (the standard deviations of the distribution across individuals are shown in Additional File 1: Figure S9). We discovered that the occurrence distributions of microbial assemblages vary from country to county; for example, Japan and Austria tend to have R-assemblages while Peru, Malawi, and Venezuela tend to have P-assemblages. Conversely, the $\mathrm{C}$-assemblage was frequently found in all countries except Japan.

\section{Correlations between microbial assemblages and butyrate-producing functions}

Dominant genera in the $\mathrm{C}$-assemblage included butyrateproducing bacteria (Table 1). Thus, we examined correlations between microbial assemblages and butyrateproducing functions (K00929: butyrate kinase, K01034: acetate CoA/acetoacetate CoA-transferase alpha subunit, and K01035: acetate CoA/acetoacetate CoA-transferase beta subunit). Figure 6 indicates the Pearson's correlation coefficients between microbial assemblages and butyrate-producing functions across individuals, showing that the $\mathrm{C}$-assemblage is significantly positively correlated with all three functions $(p<0.01$, two-sided test, after Benjamini-Hochberg correction). The P- and $\mathrm{R}$-assemblages were negatively correlated with some functions, and the B-assemblage was significantly positively correlated with only K00929, concurrent with the finding that Bacteroides fragilis has only K00929 among these three functions [35].
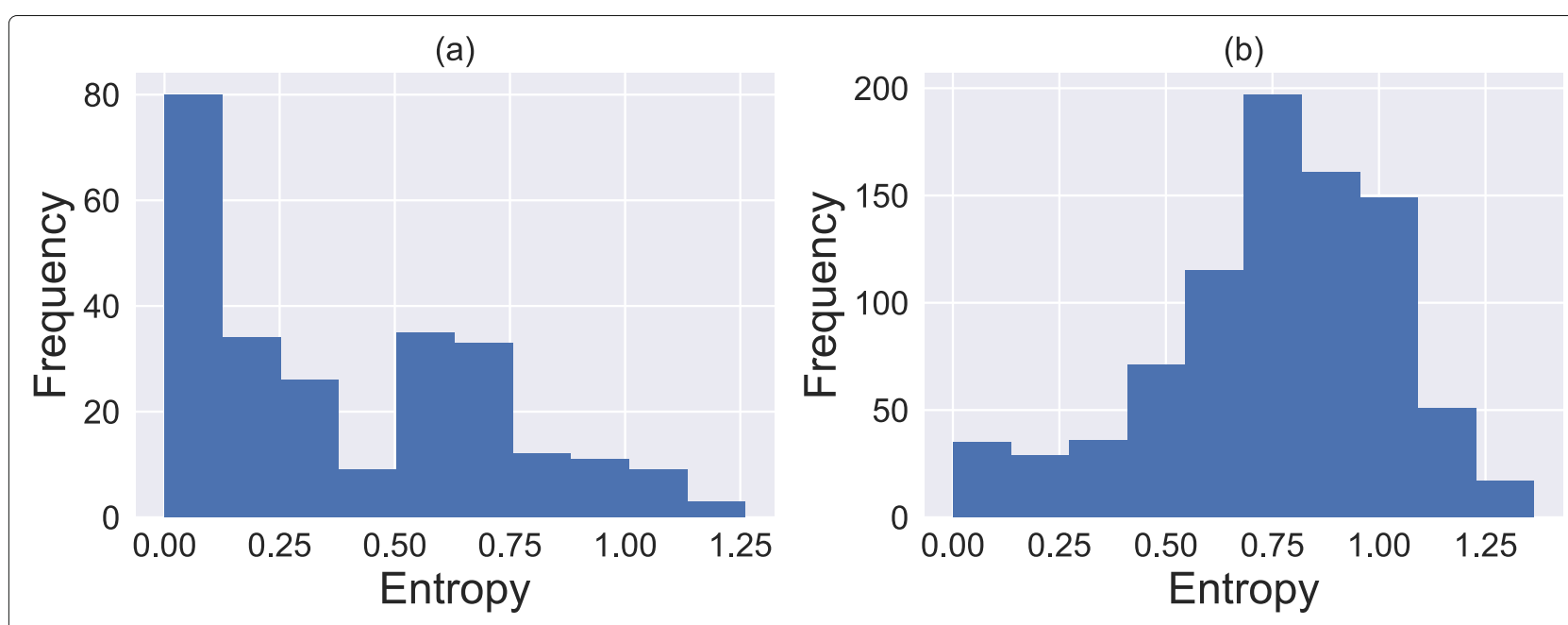

Fig. 4 Histograms of the entropy scores over assemblages a for all genera (Eq. 2) and $\mathbf{b}$ for all individuals (Eq. 1). The $x$ - and $y$-axes represent entropy and the number of samples, respectively 


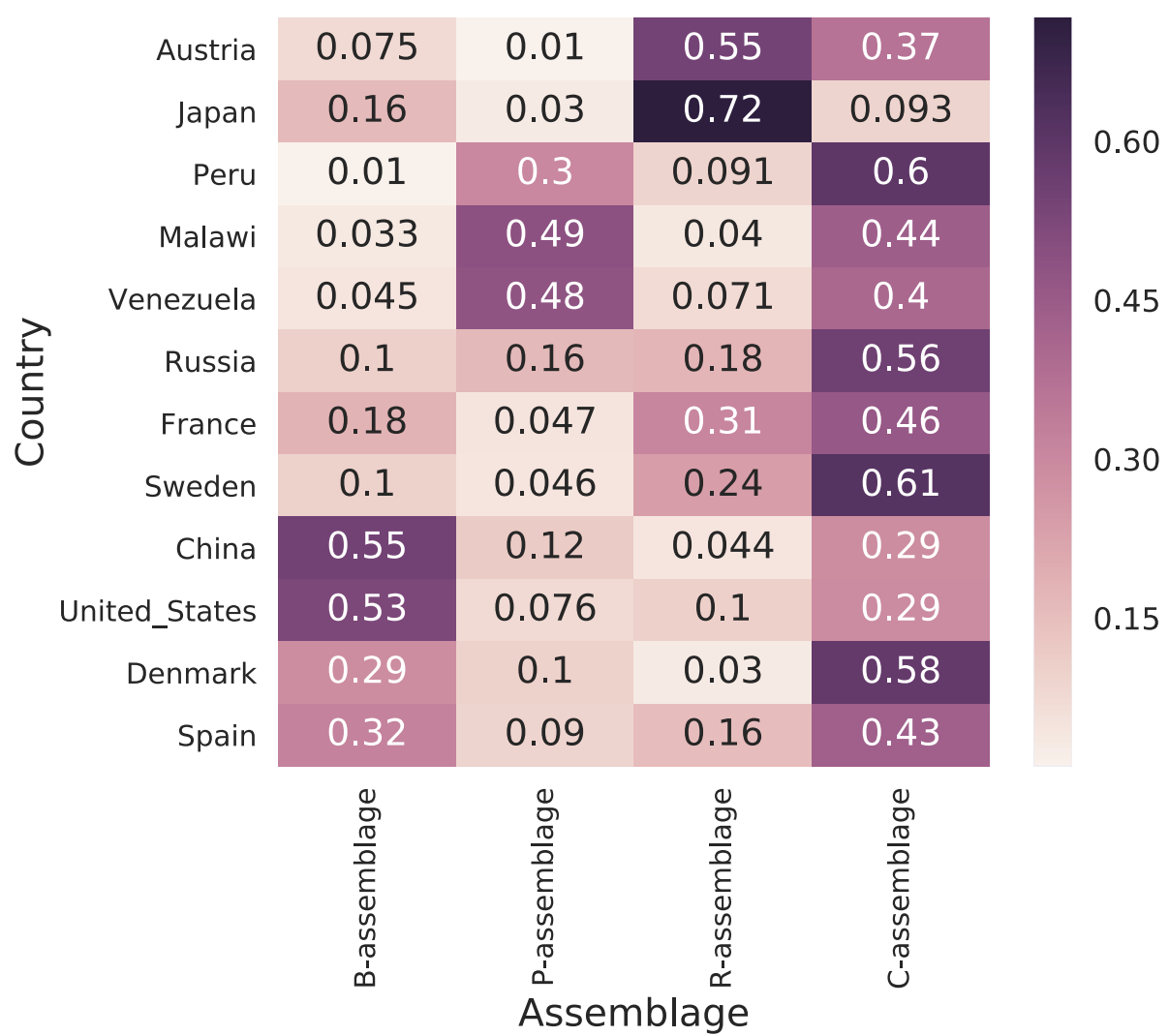

Fig. 5 Average assemblage distributions for each country. Each row shows a distribution obtained by averaging the estimated assemblage distributions across individuals of each country. The $x$ - and $y$-axes represent the microbial assemblage and country of the individual, respectively

\section{Functional profiles of each microbial assemblage}

To discuss the functional profiles of the microbial assemblages, we applied LDA to the functional profiles of individuals using the same $K$ number as for the taxonomic profiles. We regarded functional assemblages as functional profiles of the microbial assemblages. More information on this experiment is described in the supplementary section (Additional File 1: Section S1). We

Table 1 Dominant genera of the $C$-assemblage an their probabilities as estimated by LDA

\begin{tabular}{ll}
\hline Genus & Probability in C-assemblage \\
\hline Clostridium & 0.179865 \\
Eubacterium & 0.150802 \\
Unclassified Firmicutes & 0.129783 \\
Faecalibacterium & 0.093720 \\
Ruminococcus & 0.085272 \\
Roseburia & 0.074214 \\
Alistipes & 0.072359 \\
Coprococcus & 0.029497 \\
Butyrivibrio & 0.021738 \\
\hline
\end{tabular}

obtained functional assemblages with a one-to-one correspondence with the estimated microbial assemblages (Additional File 1: Figure S10). These results justify regarding functional assemblages as functional profiles of the microbial assemblages. Next, we determined the abundances of functional categories for each assemblage (Additional File 1: Figure S11) and assessed the assemblages with the largest relative abundance for each functional category (Table 2). This table shows that metabolic functions of glycan/lipid are abundant in the Bassemblage and that some specific functional categories, such as the immune system and translation, are abundant in the C-assemblage. However, Supplementary Figure S11 demonstrates that differences between assemblages are rather small.

\section{Discussion}

In this study, we used LDA for the detection of microbial assemblages in population-scale human gut microbiome data and discovered four microbial assemblages. While three assemblages (B-, P-, and R-assemblages) specifically emerged in the corresponding enterotypes (B-, P-, and R-types), the $\mathrm{C}$-assemblage was frequently observed 


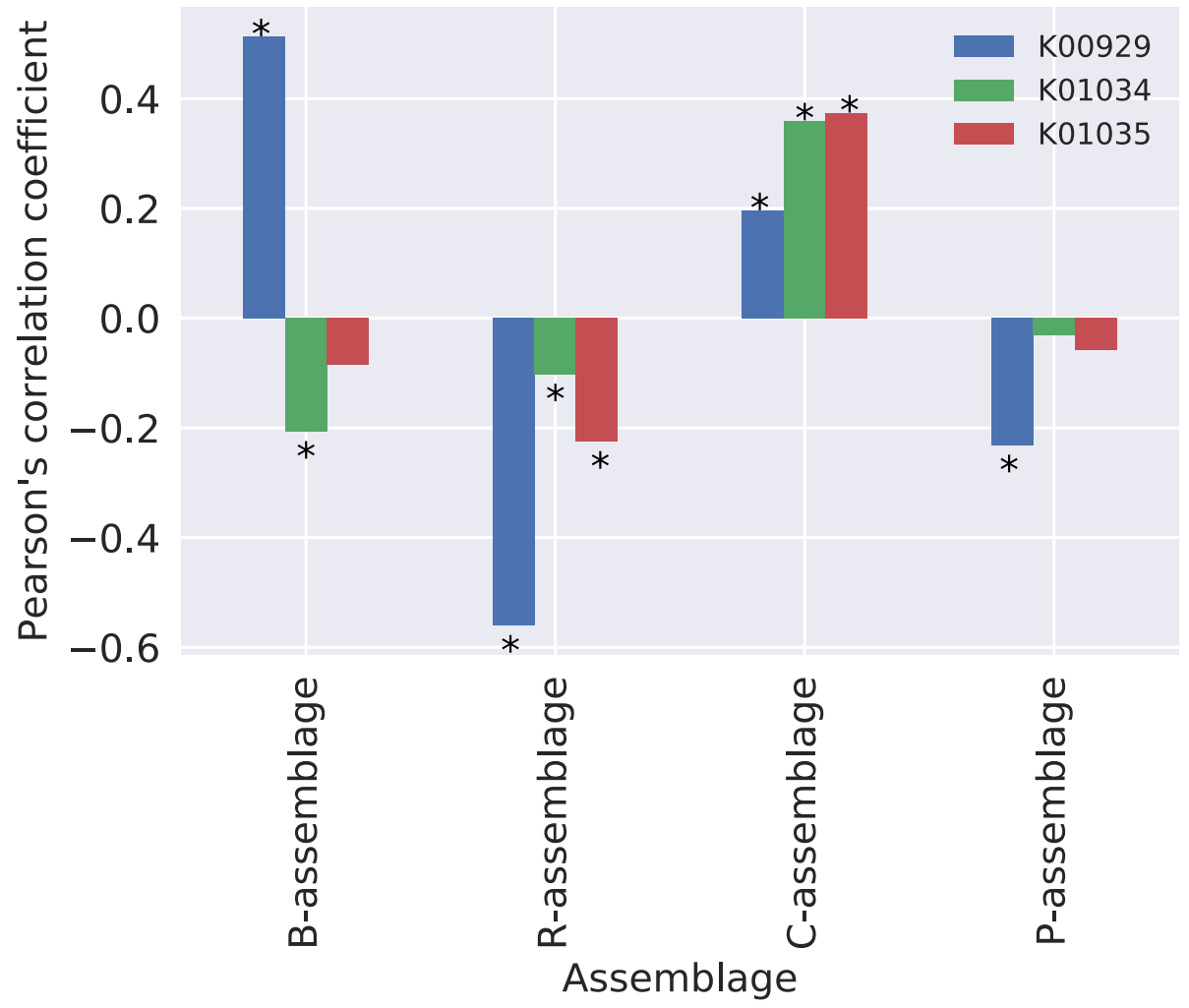

Fig. 6 The Pearson's correlation coefficients among the four assemblages and three butyrate-producing functions. The $x$ - and $y$-axes represent the assemblages and Pearson's correlation coefficients, respectively. Each bar of each assemblage indicates, from left to right, K01034, K00929, and K01035, respectively. Asterisks indicate significant differences. Significance was determined at $p<0.01$ (two-sided test, after Benjamini-Hochberg correction)

in every enterotype. As conventional cluster analysis of the samples focuses on the dominant genus of a cluster and the differences among clusters, the existence of non-dominant but shared microbial assemblages among individuals may have been overlooked. The detection of the $\mathrm{C}$-assemblage suggested that LDA is a powerful approach for revealing the assemblage structure in large metagenomic datasets.

We chose $K$, i.e., the number of assemblages, based on assemblage interpretability after comparing models with different numbers of assemblages. This task, called "model selection," is typically difficult for mixture models. Some methods for this task have been previously suggested [36, 37]. Yan et al. used cross-validation, which is a method that selects the model with the highest likelihood against the test data [19]. However, these methods tend to overestimate $K$, leading to difficulties in clarifying the association between enterotypes and assemblages. Indeed, Yan et al. estimated $K=60$, although the number of samples was less than in this study.

As the model we used is rather simple (that is, $K$ is small), it might fail to adequately capture the structure of the data. Hence, we confirmed whether our results were consistent with the data in two ways. First, we verified that the relative abundance (Additional File 1: Figure S12) was consistent with the estimated assemblage distribution (Fig. 2c). Each genus was regarded as mainly appearing in the assemblage with the highest $P(k \mid j)$, as defined by Eq. 3. These results were consistent with the estimated parameters shown in Fig. 2c. Second, we verified that most genera within the same assemblage were significantly positively correlated across samples based on the Spearman's correlation coefficients across samples between the major genera of the $\mathrm{B}_{-}, \mathrm{P}_{-}, \mathrm{R}_{-}$, and $\mathrm{C}$-assemblages $(p<0.01$, two-sided test, after BenjaminiHochberg correction [38], Additional File 1: Figure S13). Some genera (i.e., Eubacterium, Faecalibacterium, Dorea, Ruminococcus, Streptococcus, and Catenibacterium) were significantly positively correlated with many genera in other assemblages. These results are in agreement with the fact that their $P(k \mid j)$ is high for multiple assemblages (Additional File 1: Figure S14). For example, Ruminococcus has a positive correlation with the genera mainly appearing in the R-assemblage. Indeed, Ruminococcus has a high association with the $\mathrm{R}$-assemblage even though its main assemblage is the $\mathrm{C}$-assemblage. 
Table 2 Functional assemblage having the largest relative abundance for each functional category

\begin{tabular}{|c|c|}
\hline Functional category & Functional assemblage \\
\hline Biosynthesis of other secondary metabolites & B-assemblage(ko) \\
\hline Carbohydrate metabolism & B-assemblage(ko) \\
\hline Lipid metabolism & B-assemblage(ko) \\
\hline Transport and catabolism & B-assemblage(ko) \\
\hline Digestive system & B-assemblage(ko) \\
\hline Endocrine system & B-assemblage(ko) \\
\hline Glycan biosynthesis and metabolism & B-assemblage(ko) \\
\hline Environmental adaptation & B-assemblage(ko) \\
\hline Energy metabolism & P-assemblage(ko) \\
\hline Endocrine and metabolic diseases & P-assemblage(ko) \\
\hline Immune diseases & P-assemblage(ko) \\
\hline Infectious diseases & P-assemblage(ko) \\
\hline Metabolism of other amino acids & P-assemblage(ko) \\
\hline Metabolism of terpenoids and polyketides & P-assemblage(ko) \\
\hline Nervous system & P-assemblage(ko) \\
\hline Excretory system & P-assemblage(ko) \\
\hline Folding, sorting, and degradation & P-assemblage(ko) \\
\hline Transcription & R-assemblage(ko) \\
\hline Amino acid metabolism & R-assemblage(ko) \\
\hline Metabolism of cofactors and vitamins & R-assemblage(ko) \\
\hline Membrane transport & R-assemblage(ko) \\
\hline Cell communication & R-assemblage(ko) \\
\hline Signaling molecules and interaction & R-assemblage(ko) \\
\hline Xenobiotics biodegradation and metabolism & R-assemblage(ko) \\
\hline Immune system & C-assemblage(ko) \\
\hline Nucleotide metabolism & C-assemblage(ko) \\
\hline Neurodegenerative diseases & C-assemblage(ko) \\
\hline Substance dependence & C-assemblage(ko) \\
\hline Replication and repair & C-assemblage(ko) \\
\hline Signal transduction & C-assemblage(ko) \\
\hline Cell motility & C-assemblage(ko) \\
\hline Cell growth and death & C-assemblage(ko) \\
\hline Translation & C-assemblage(ko) \\
\hline Cardiovascular diseases & C-assemblage (ko) \\
\hline Cancers & C-assemblage(ko) \\
\hline
\end{tabular}

Each functional assemblage is indicated by the name of the corresponding microbial assemblage with (ko) appended

As mentioned earlier, the genera mainly appearing in the $\mathrm{B}$ - and $\mathrm{P}$-assemblages tend to occur in the $\mathrm{B}$ - and P-types, respectively. The genera specifically appearing in the B- and P-types were reported to have functions for metabolizing protein/animal fat and carbohydrates, respectively [29], and the genera mainly appearing in the
B- and P-assemblages may consequently have the same functions. We could confirm that lipid metabolism functions were abundant in the B-assemblage through functional assemblage analysis. This result suggests that the Bassemblage in the human gut becomes dominant through a fat-rich diet. Similarly, the genera mainly appearing in the $\mathrm{C}$-assemblage may have functions that do not correspond with dietary habits because they appeared in all enterotypes. This suggestion is concurrent with the finding that functions related to immune cells and translation are abundant in the $\mathrm{C}$-assemblage. The assemblage distributions for each country also suggests a relationship between dietary habits and assemblage. Peru, Malawi, and Venezuela, where staple foods include corn, have high Passemblage abundance. We could not establish a similarity in dietary habits between Japan and Austria though their distributions are similar.

The noticeable characteristic of Japan, i.e., low Cassemblage abundance, was observed. Nishijima et al. reported that the Japanese gut microbiome is characterized by the low abundance of Clostridium and unclassified Firmicutes, which are the main components of the Cassemblage (Table 1) based on the same dataset [28]. Japan has the highest abundance of the R-assemblage, which shares Ruminococcus and Eubacterium with the $\mathrm{C}$-assemblage. Hence, the two assemblages may have similar metabolic functions. Incidentally, Eubacterium and Faecalibacterium, which are the abundant genera in the Cassemblage, were not less abundant in the Japanese population compared with that of other countries (Additional File 1: Figure S15).

There are two interesting points regarding the Cassemblage. First, it can coexist with all of the other three assemblages, which were found in almost all countries. Therefore, the genera mainly appearing in the Cassemblage are generalists in the human gut environment $[39,40]$. While generalists can adapt to diverse environments, they are not specialized to particular environments unlike specialists. This difference in survival strategy may be the reason why the genera mainly appearing in the Cassemblage were not dominant in the human gut microbiome. It is therefore possible that the $\mathrm{C}$-assemblage is the core gut microbiome $[9,41]$. However, $\mathrm{C}$-assemblage abundance is not consistent from person to person; as such, what determines the existence of $\mathrm{C}$-assemblages in the gut microbiome is unclear. Second, the dominant genera of the C-assemblage (such as Clostridium, Eubacterium, Faecalibacterium, Roseburia, Coprococcus, and Butyrivibrio) include representative butyrate-producing species (Table 1) [42, 43]. In addition, we found that the Cassemblage correlates with the three butyrate-producing functions. Butyrate is known to have anti-inflammatory effects [44] and is associated with IBD, type-2 diabetes, and colorectal cancer [45-47]. Therefore, C-assemblage 
abundance may indicate the health of its hosts, although the dataset used in this study contained only healthy individuals. In addition, we found that the ages and body mass indices (BMIs) of hosts did not relate to the presence of the $\mathrm{C}$-assemblage (Additional File 1: Figure S16). Further research is accordingly required, such as via comparisons of $\mathrm{C}$-assemblage abundance between individuals with and without a disease.

We envision two future directions for applications of LDA to metagenomic data. The first is its application to more diverse datasets. Metagenomic data have been sampled from not only human guts but also various other environments, such as the atmosphere [48], ocean $[49,50]$, and soil [51]. Application of LDA to these data should help reveal the structure of microbial assemblages on a global scale [52]. For example, Sommeria-Klein et al. recently applied LDA to taxonomic profiles of a tropical forest soil DNA dataset to reveal spatial structures [53]. The second direction is the extension of the LDA model-LDA has high model extensibility. Indeed, many extended LDA models have been proposed for naturallanguage processing [54-57]. The application of these extended LDA models to metagenomic analysis is a fascinating research focus for further elucidation of microbial assemblage structure. For example, applying supervised topic models [58], which utilize label information to estimate assemblage structures, to patient metagenomic data could detect microbial assemblages related to disease. The pachinko allocation model [54], which models hierarchical assemblage structures, may be useful for revealing sub-assemblages within an assemblage. A transition in assemblage composition can be estimated from timeseries data from the human gut microbiome [59] using the topic tracking model [57].

\section{Conclusions}

In this study, we conducted a microbial assemblage analysis on a large-scale human gut metagenome dataset using LDA. We discovered that three assemblages specifically emerged in the corresponding enterotypes, but the $\mathrm{C}$-assemblage was frequently observed in all three enterotypes. In addition, we revealed that the dominant genera of the $\mathrm{C}$-assemblage include representative butyrate-producing species. Further elucidation of the function of the $\mathrm{C}$-assemblage or investigation of the relationship between disease and the $\mathrm{C}$-assemblage is an important research direction.

\section{Supplementary information}

Supplementary information accompanies this paper at https://doi.org/10.1186/s40168-020-00864-3.

Additional file 1: This file includes Section S1, Figures S1, S2, S3, S4, S5, S6 S7, S8, S9, S10, S11, S12, S13, S14, S15, S16, and Table S1.

\section{Abbreviations}

LDA: Latent Dirichlet allocation; IBD: Inflammatory bowel disease; PAM: Partitioning around medoids; DMM: Dirichlet multinomial mixture; BMI: Body mass index; VB: Variational Bayes; VLB: Variational lower-bound

\section{Acknowledgements}

The computations in this research were performed using the supercomputing facilities at the National Institute of Genetics in Research Organization of Information and Systems. We thank anonymous reviewers for their constructive and insightful comments, which greatly improved our manuscript.

\section{Authors' contributions}

M. Hamada and TF conceived the study. M. Hamada supervised this study. SN and M. Hattori processed the data. SH implemented the method and performed all the computational experiments. SH, SN, TF, and M. Hamada analyzed the results. TF, SH, and M. Hamada wrote the draft manuscript, and $\mathrm{SN}$ and $\mathrm{M}$. Hattori critically revised the manuscript. All authors read and approved the final manuscript.

\section{Funding}

This work was supported by the Ministry of Education, Culture, Sports, Science, and Technology (KAKENHI) (grant numbers JP16H05879, JP16H01318, JP16H02484, and 17K20032 to MH, JP16H06279).

\section{Availability of data and materials}

Supplementary material is available from the journal website. The implementation of the algorithm is available at GitHub(https://github.com/ shion-h/TopicModels and https://github.com/shion-h/

PartitionAroundMedoids).

\section{Ethics approval and consent to participate}

Not applicable.

\section{Consent for publication}

Not applicable.

\section{Competing interests}

The authors declare that they have no competing interests.

\section{Author details}

${ }^{1}$ Graduate School of Advanced Science and Engineering, Waseda University, 55N-06-10, 3-4-1, Okubo Shinjuku-ku, 169-8555 Tokyo, Japan. ${ }^{2}$ Computational Bio Big-Data Open Innovation Laboratory (CBBD-OIL), National Institute of Advanced Industrial Science and Technology (AIST), Tokyo, Japan.

${ }^{3}$ Computational Biology and Medical Sciences, Graduate School of Frontier Sciences, The University of Tokyo, Chiba, Japan. ${ }^{4}$ Department of Computer Science, Graduate School of Information Science and Engineering, The University of Tokyo, Tokyo, Japan. ${ }^{5}$ RIKEN Center for Integrative Medical Sciences, Kanagawa, Japan. ${ }^{6}$ Graduate School of Medicine, Nippon Medical School, Tokyo, Japan. ${ }^{7}$ Center for Data Science, Waseda University, Tokyo, Japan.

Received: 8 May 2018 Accepted: 13 May 2020

Published online: 23 June 2020

\section{References}

1. Kurokawa K, Itoh T, Kuwahara T, Oshima K, Toh H, Toyoda A, Takami H, Morita H, Sharma VK, Srivastava TP, Taylor TD, Noguchi H, Mori H, Ogura Y, Ehrlich DS, Itoh K, Takagi T, Sakaki Y, Hayashi T, Hattori M. Comparative Metagenomics Revealed Commonly Enriched Gene Sets in Human Gut Microbiomes. DNA Res. 2007;14(4):169-81. https://doi.org/10. 1093/dnares/dsm018.

2. Mai V, McCrary QM, Sinha R, Glei M. Associations between dietary habits and body mass index with gut microbiota composition and fecal water genotoxicity: an observational study in African American and Caucasian American volunteers. Nutr J. 2009;8:49. https://doi.org/10.1186/14752891-8-49.

3. Cho I, Blaser MJ. The human microbiome: at the interface of health and disease. Nat Rev Genet. 2012;13(4):260-70. https://doi.org/10.1038/ nrg3182. 
4. Xavier RJ, Podolsky DK. Unravelling the pathogenesis of inflammatory bowel disease. Nature. 2007;448(7152):427-34. https://doi.org/10.1038/ nature06005.

5. Giongo A, Gano KA, Crabb DB, Mukherjee N, Novelo LL, Casella G, Drew JC, Ilonen J, Knip M, Hyöty H, Veijola R, Simell T, Simell O, Neu J, Wasserfall CH, Schatz D, Atkinson MA, Triplett EW. Toward defining the autoimmune microbiome for type 1 diabetes. ISME J. 2011;5(1):82-91. https://doi.org/10.1038/ismej.2010.92.

6. Kostic AD, Gevers D, Pedamallu CS, Michaud M, Duke F, Earl AM, Ojesina Al, Jung J, Bass AJ, Tabernero J, Baselga J, Liu C, Shivdasani RA, Ogino S, Birren BW, Huttenhower C, Garrett WS, Meyerson M. Genomic analysis identifies association of Fusobacterium with colorectal carcinoma. Genome Res. 2012;22(2):292-8. https://doi.org/10.1101/gr.126573.111.

7. Mulle JG, Sharp WG, Cubells JF. The gut microbiome: a new frontier in autism research. Curr Psychiatr Rep. 2013;15(2):337. https://doi.org/10. 1007/s11920-012-0337-0.

8. Clemente JC, Ursell LK, Parfrey LW, Knight R. The impact of the gut microbiota on human health: An integrative view. Cell. 2012;148(6): 1258-70. https://doi.org/10.1016/j.cell.2012.01.035

9. Turnbaugh PJ, Hamady M, Yatsunenko T, Cantarel BL, Duncan A, Ley RE, Sogin ML, Jones WJ, Roe BA, Affourtit JP, Egholm M, Henrissat B, Heath $A C$, Knight R, Gordon II. A core gut microbiome in obese and lean twins. Nature. 2009;457(7228):480-4. https://doi.org/10.1038/nature07540.

10. Qin J, Li R, Raes J, Arumugam M, Burgdorf KS, Manichanh C, Nielsen T, Pons N, Levenez F, Yamada T, Mende DR, Li J, Xu J, Li S, Li D, Cao J, Wang B, Liang $H$, Zheng $H$, Xie $Y$, Tap J, Lepage $P$, Bertalan M, Batto J-M, Hansen T, Paslier DL, Linneberg A, Nielsen HB, Pelletier E, Renault P, Sicheritz-Ponten T, Turner K, Zhu H, Yu C, Li S, Jian M, Zhou Y, Li Y, Zhang X, Li S, Qin N, Yang H, Wang J, Brunak S, Doré J, Guarner F, Kristiansen K, Pedersen O, Parkhill J, Weissenbach J, Consortium M, Antolin M, Artiguenave F, Blottiere H, Borruel N, Bruls T, Casellas F, Chervaux C, Cultrone A, Delorme C, Denariaz G, Dervyn R, Forte M, Friss $C$, van de Guchte $M$, Guedon E, Haimet F, Jamet A, Juste C, Kaci G, Kleerebezem M, Knol J, Kristensen M, Layec S, Roux KL, Leclerc M, Maguin E, Minardi RM, Oozeer R, Rescigno M, Sanchez N, Tims S, Torrejon $T$, Varela $E$, de Vos W, Winogradsky $Y$, Zoetendal E, Bork P, Ehrlich SD, Wang J. A human gut microbial gene catalogue established by metagenomic sequencing. Nature. 2010;464(7285):59-65. https://doi. org/10.1038/nature08821.

11. Yatsunenko T, Rey FE, Manary MJ, Trehan I, Dominguez-Bello MG, Contreras M, Magris M, Hidalgo G, Baldassano RN, Anokhin AP, Heath AC, Warner B, Reeder J, Kuczynski J, Caporaso JG, Lozupone CA, Lauber C, Clemente JC, Knights D, Knight R, Gordon JI. Human gut microbiome viewed across age and geography. Nature. 2012;486(7402):222-7. https:// doi.org/10.1038/nature11053.

12. Arumugam M, Raes J, Pelletier E, Paslier DL, Yamada T, Mende DR, Fernandes GR, Tap J, Bruls T, Batto J-M, Bertalan M, Borruel N, Casellas F, Fernandez L, Gautier L, Hansen T, Hattori M, Hayashi T, Kleerebezem M, Kurokawa K, Leclerc M, Levenez F, Manichanh C, Nielsen HB, Nielsen T, Pons N, Poulain J, Qin J, Sicheritz-Ponten T, Tims S, Torrents D, Ugarte E, Zoetendal EG, Wang J, Guarner F, Pedersen O, de Vos WM, Brunak S, Doré J, Members MCa, Antolín M, Artiguenave F, Blottiere HM, Almeida M, Brechot C, Cara C, Chervaux C, Cultrone A, Delorme C, Denariaz G, Dervyn R, Foerstner KU, Friss C, van de Guchte M, Guedon E, Haimet F, Huber W, van Hylckama-Vlieg J, Jamet A, Juste C, Kaci G, Knol J, Kristiansen K, Lakhdari O, Layec S, Roux KL, Maguin E, Mérieux A Minardi RM, M'rini C, Muller J, Oozeer R, Parkhill J, Renault P, Rescigno M, Sanchez N, Sunagawa S, Torrejon A, Turner K, Vandemeulebrouck G, Varela E, Winogradsky Y, Zeller G, Weissenbach J, Ehrlich SD, Bork P. Enterotypes of the human gut microbiome. Nature. 2011;473(7346): 174-80. https://doi.org/10.1038/nature09944.

13. Ding T, Schloss PD. Dynamics and associations of microbial community types across the human body. Nature. 2014;509(7500):357-60. https:// doi.org/10.1038/nature13178.

14. Holmes I, Harris K, Quince C. Dirichlet multinomial mixtures: generative models for microbial metagenomics. PLoS ONE. 2012;7(2):30126. https:// doi.org/10.1371/journal.pone.0030126.

15. Shafiei M, Dunn KA, Boon E, MacDonald SM, Walsh DA, Gu H, Bielawski JP. BioMiCo: a supervised Bayesian model for inference of microbial community structure. Microbiome. 2015;3:8. https://doi.org/10.1186/ s40168-015-0073-x.
16. Boon E, Meehan CJ, Whidden C, Wong DH-J, Langille MGI, Beiko RG. Interactions in the microbiome: communities of organisms and communities of genes. FEMS Microbiol Rev. 2014;38(1):90-118. https:// doi.org/10.1111/1574-6976.12035

17. Cai Y, Gu H, Kenney T. Learning microbial community structures with supervised and unsupervised non-negative matrix factorization. Microbiome. 2017;5:110. https://doi.org/10.1186/s40168-017-0323-1.

18. Higashi K, Suzuki S, Kurosawa S, Mori H, Kurokawa K. Latent environment allocation of microbial community data. PLOS Comput Biol. 2018;14(6):1006143. https://doi.org/10.1371/journal.pcbi.1006143.

19. Yan J, Chuai G, Qi T, Shao F, Zhou C, Zhu C, Yang J, Yu Y, Shi C, Kang N, He Y, Liu Q. MetaTopics: an integration tool to analyze microbial community profile by topic model. BMC Genomics. 2017;18(1):962. https://doi.org/10.1186/s12864-016-3257-2.

20. Sankaran K, Holmes SP. Latent variable modeling for the microbiome. Biostatistics. 2019;20(4):599-614. https://doi.org/10.1093/biostatistics/ kxy018.

21. Knights D, Kuczynski J, Charlson ES, Zaneveld J, Mozer MC, Collman RG, Bushman FD, Knight R, Kelley ST. Bayesian community-wide culture-independent microbial source tracking. Nat Methods. 2011;8(9): 761-3. https://doi.org/10.1038/nmeth.1650.

22. Shafiei M, Dunn KA, Chipman H, Gu H, Bielawski JP. BiomeNet: a Bayesian model for inference of metabolic divergence among microbial communities. PLOS Comput Biol. 2014;10(11):1003918. https://doi.org/10 1371/journal.pcbi.1003918.

23. Blei DM, Ng AY, Jordan MI. Latent Dirichlet Allocation. J Mach Learn Res. 2003;3(Jan):993-1022.

24. Liu B, Liu L, Tsykin A, Goodall GJ, Green JE, Zhu M, Kim CH, Li J. Identifying functional miRNA-mRNA regulatory modules with correspondence latent Dirichlet allocation. Bioinformatics. 2010;26(24): 3105-11. https://doi.org/10.1093/bioinformatics/btq576.

25. Wu Y, Liu M, Zheng WJ, Zhao Z, Xu H. Ranking gene-drug relationships in biomedical literature using latent Dirichlet allocation. World Sci. 2012. https://doi.org/10.1142/9789814366496_0041.

26. Pinoli P, Chicco D, Masseroli M. Latent Dirichlet allocation based on Gibbs sampling for gene function prediction. In: 2014 IEEE Conference on Computational Intelligence in Bioinformatics and Computational Biology; 2014. p. 1-8. https://doi.org/10.1109/CIBCB.2014.6845514.

27. Chen $X, H e T$, Hu X, An Y, Wu X. Inferring functional groups from microbial gene catalogue with probabilistic topic models. In: 2011 IEEE International Conference on Bioinformatics and Biomedicine; 2011. p. 3-9. https://doi.org/10.1109/BIBM.2011.12.

28. Nishijima S, Suda W, Oshima K, Kim S-W, Hirose Y, Morita H, Hattori M. The gut microbiome of healthy Japanese and its microbial and functional uniqueness. DNA Res Int J Rapid Publ Rep Genes Genomes. 2016;23(2): 125-33. https://doi.org/10.1093/dnares/dsw002.

29. Wu GD, Chen J, Hoffmann C, Bittinger K, Chen Y-Y, Keilbaugh SA, Bewtra M, Knights D, Walters WA, Knight R, Sinha R, Gilroy E, Gupta K, Baldassano R, Nessel L, Li H, Bushman FD, Lewis JD. Linking long-term dietary patterns with gut microbial enterotypes. Science. 2011;334(6052): 105-8. https://doi.org/10.1126/science.1208344.

30. Kanehisa M, Goto S. KEGG: Kyoto Encyclopedia of Genes and Genomes. Nucleic Acids Res. 2000;28(1):27-30. https://doi.org/10.1093/nar/28.1.27.

31. Rousseeuw PJ. Silhouettes: a graphical aid to the interpretation and validation of cluster analysis. J Comput Appl Math. 1987;20:53-65. https:// doi.org/10.1016/0377-0427(87)90125-7.

32. Asuncion A, Welling M, Smyth P, Teh YW. On smoothing and inference for topic models. In: Proceedings of the Twenty-Fifth Conference on Uncertainty in Artificial Intelligence, UAl'09. Montreal, Quebec, Canada: AUAI Press; 2009. p. 27-34.

33. Minka T. Estimating a Dirichlet distribution. Technical report, MIT. 2000.

34. Wallach HM, Mimno DM, McCallum A. Rethinking LDA: why priors matter. Adv Neural Inf Process Syst. 2009;22:1973-81.

35. KO (KEGG ORTHOLOGY) Database. https://www.kegg.jp/kegg/ko.html. Accessed 28 Feb 2019.

36. Corduneanu A, Bishop CM. Variational Bayesian model selection for mixture distributions. MA: Morgan Kaufmann Waltham; 2001.

37. Fujimaki R, Morinaga S. Factorized asymptotic Bayesian inference for mixture modeling. In: Artificial Intelligence and Statistics; 2012. p. 400-8.

38. Benjamini $Y$, Hochberg Y. Controlling the false discovery rate: a practical and powerful approach to multiple testing. J R Stat Soc Ser B Methodol. 1995;57(1):289-300. 
39. Pandit SN, Jurek K, Karl C. Contrasts between habitat generalists and specialists: an empirical extension to the basic metacommunity framework. Ecology. 2009;90(8):2253-62. https://doi.org/10.1890/080851.1.

40. Sriswasdi S, Yang C.-C., Iwasaki W. Generalist species drive microbial dispersion and evolution. Nat Commun. 2017;8(1):1162. https://doi.org/ 10.1038/s41467-017-01265-1.

41. Turnbaugh PJ, Gordon Jl. The core gut microbiome, energy balance and obesity. J Physiol. 2009;587(Pt 17):4153-8. https://doi.org/10.1113/ jphysiol.2009.174136.

42. Pryde SE, Duncan SH, Hold GL, Stewart CS, Flint HJ. The microbiology of butyrate formation in the human colon. FEMS Microbiol Lett. 2002;217(2): 133-9.

43. Louis P, Flint HJ. Diversity, metabolism and microbial ecology of butyrateproducing bacteria from the human large intestine. FEMS Microbiol Lett. 2009;294(1):1-8. https://doi.org/10.1111/j.1574-6968.2009.01514.x.

44. Sokol H, Pigneur B, Watterlot L, Lakhdari $\mathrm{O}$, Bermúdez-Humarán LG, Gratadoux J-J, Blugeon S, Bridonneau C, Furet J-P, Corthier G, Grangette C, Vasquez N, Pochart $P$, Trugnan $G$, Thomas G, Blottière HM, Doré J, Marteau P, Seksik P, Langella P. Faecalibacterium prausnitzii is an anti-inflammatory commensal bacterium identified by gut microbiota analysis of Crohn disease patients. Proc Natl Acad Sci U S A. 2008;105(43): 16731-6. https://doi.org/10.1073/pnas.0804812105.

45. Furusawa Y, Obata Y, Fukuda S, Endo TA, Nakato G, Takahashi D, Nakanishi Y, Uetake C, Kato K, Kato T, Takahashi M, Fukuda NN, Murakami S, Miyauchi E, Hino S, Atarashi K, Onawa S, Fujimura Y, Lockett T, Clarke JM, Topping DL, Tomita M, Hori S, Ohara O, Morita T, Koseki H, Kikuchi J, Honda K, Hase K, Ohno H. Commensal microbederived butyrate induces the differentiation of colonic regulatory $T$ cells. Nature. 2013;504(7480):446-50. https://doi.org/10.1038/nature12721.

46. Nicholson JK, Holmes E, Kinross J, Burcelin R, Gibson G, Jia W, Pettersson S. Host-Gut microbiota metabolic interactions. Science. 2012;336(6086):1262-7. https://doi.org/10.1126/science.1223813.

47. Tremaroli $V$, Bäckhed F. Functional interactions between the gut microbiota and host metabolism. Nature. 2012;489(7415):242-9. https:// doi.org/10.1038/nature11552.

48. Tringe SG, Zhang T, Liu X, Yu Y, Lee WH, Yap J, Yao F, Suan ST, Ing SK, Haynes M, Rohwer F, Wei CL, Tan P, Bristow J, Rubin EM, Ruan Y. The airborne metagenome in an indoor urban environment. PLoS ONE. 2008;3(4):1862. https://doi.org/10.1371/journal.pone.0001862.

49. Venter JC, Remington $\mathrm{K}$, Heidelberg JF, Halpern AL, Rusch D, Eisen JA, Wu D, Paulsen I, Nelson KE, Nelson W, Fouts DE, Levy S, Knap AH, Lomas MW, Nealson K, White O, Peterson J, Hoffman J, Parsons R, Baden-Tillson H, Pfannkoch C, Rogers Y-H, Smith HO. Environmental genome shotgun sequencing of the Sargasso Sea. Science. 2004;304(5667):66-74. https://doi.org/10.1126/science.1093857.

50. Sunagawa S, Coelho LP, Chaffron S, Kultima JR, Labadie K, Salazar G, Djahanschiri B, Zeller G, Mende DR, Alberti A, Cornejo-Castillo FM, Costea PI, Cruaud C, d'Ovidio F, Engelen S, Ferrera I, Gasol JM, Guidi L, Hildebrand F, Kokoszka F, Lepoivre C, Lima-Mendez G, Poulain J, Poulos BT, Royo-Llonch M, Sarmento H, Vieira-Silva S, Dimier C, Picheral M, Searson S, Kandels-Lewis S, Coordinators TO, Bowler C, Vargas C. d. Gorsky G, Grimsley N, Hingamp P, ludicone D, Jaillon O, Not F, Ogata H, Pesant S, Speich S, Stemmann L, Sullivan MB, Weissenbach J, Wincker $P$, Karsenti E, Raes J, Acinas SG, Bork P. Structure and function of the global ocean microbiome. Science. 2015;348(6237):. https://doi.org/10. 1126/science.1261359.

51. Fierer N, Leff JW, Adams BJ, Nielsen UN, Bates ST, Lauber CL, Owens S, Gilbert JA, Wall DH, Caporaso JG. Cross-biome metagenomic analyses of soil microbial communities and their functional attributes. Proc Natl Acad Sci. 2012;109(52):21390-5. https://doi.org/10.1073/pnas.1215210110.

52. Chaffron S, Rehrauer H, Pernthaler J, von Mering C. A global network of coexisting microbes from environmental and whole-genome sequence data. Genome Res. 2010;20(7):947-59. https://doi.org/10.1101/gr.104521. 109.

53. Sommeria-Klein $G$, Zinger $L$, Coissac $E$, Iribar $A$, Schimann $H$, Taberlet $P$, Chave J. Latent Dirichlet allocation reveals spatial and taxonomic structure in a DNA-based census of soil biodiversity from a tropical forest. Mol Ecol Resour. 2019;n/a(n/a):. https://doi.org/10.1111/1755-0998.13109.

54. Li W, McCallum A. Pachinko allocation: DAG-structured mixture models of topic correlations. In: Proceedings of the 23rd International Conference on Machine Learning, ICML '06. New York, NY, USA: ACM; 2006. p. 577-84. https://doi.org/10.1145/1143844.1143917.

55. Lacoste-Julien S, Sha F, Jordan MI. DiscLDA: discriminative learning for dimensionality reduction and classification. Adv Neural Inf Process Syst. 2009;21:897-904.

56. Ramage D, Hall D, Nallapati R, Manning CD. Labeled LDA: a supervised topic model for credit attribution in multi-labeled corpora. In: Proceedings of the 2009 Conference on Empirical Methods in Natural Language Processing: Volume 1 - Volume 1, EMNLP '09. Stroudsburg, PA, USA: Association for Computational Linguistics; 2009. p. 248-256.

57. Iwata T, Watanabe S, Yamada T, Ueda N. Topic tracking model for analyzing consumer purchase behavior. In: Proceedings of the $21 \mathrm{st}$ International Jont Conference on Artifical Intelligence, IJCAI'09. San Francisco, CA, USA: Morgan Kaufmann Publishers Inc.; 2009. p. 1427-32.

58. Mcauliffe JD, Blei DM. Supervised topic models. Adv Neural Inf Process Syst. 2008;20:121-8.

59. David LA, Materna AC, Friedman J, Campos-Baptista MI, Blackburn MC, Perrotta A, Erdman SE, Alm EJ. Host lifestyle affects human microbiota on daily timescales. Genome Biol. 2014;15:89. https://doi.org/10.1186/gb2014-15-7-r89.

\section{Publisher's Note}

Springer Nature remains neutral with regard to jurisdictional claims in published maps and institutional affiliations.
Ready to submit your research? Choose BMC and benefit from:

- fast, convenient online submission

- thorough peer review by experienced researchers in your field

- rapid publication on acceptance

- support for research data, including large and complex data types

- gold Open Access which fosters wider collaboration and increased citations

- maximum visibility for your research: over $100 \mathrm{M}$ website views per yea

At $B M C$, research is always in progress.

Learn more biomedcentral.com/submission 